\title{
On a M/G/1 Queue Subject to Random Breakdowns with General Repair Times and Server's Choice for Deterministic or General Server Vacations
}

\author{
Kailash C. Madan \\ Department of Mathematical Sciences, Ahlia University, Bahrain \\ Correspondence Author: Kailash C. Madan, Department of Mathematical Sciences, Ahlia University, Bahrain
}

Received date: 22 April 2019, Accepted date: 22 July 2019, Online date: 25 August 2019

Copyright: (c) 2019 Kailash C. Madan.2019. This is an open-access article distributed under the terms of the Creative Commons Attribution License, which permits unrestricted use, distribution, and reproduction in any medium, provided the original author and source are cre dited.

\begin{abstract}
We study an M/G/1 Type queueing system subject to random failures. The customers arrive at the system in a Poisson stream following a compound Poisson process and are server oen by one under the FIFO service discipline. On occurrence of a failure the repairs on the service channel start instantly. The system starts working as soon the repairs are complete. In addition, the server may take a vacation after completing every service. If the server chooses to take a vacation, he has the option of taking a deterministic vacation of constant duration or a general vacation of random length of time. Following the generating function approach, we find interesting steady state results in clear and discrete form.
\end{abstract}

Keywords: Poisson arrivals, general service, random breakdowns, deterministic vacation, general vacation, steady state

\section{INTRODUCTION}

Almost all queuing systems in real life are subject to sudden breakdown or server vacations in case of human servers. Such breakdowns and server vacations adversely affect the system efficiency and customers waiting time. Many research papers have come up in the literature of queues on such failures and vacations. We want to refer to Gaver [1], Takagi [5], Tegham [6] and Madan [2, 3, 4].

In the present work, we deal with an M/G/1 queueing system which is subject to sudden random failures. As soon as failure occurs, the server instantly goes under repairs following a general repair time distribution. Besides, we assume that immediately after completion of service, the server has the option to take a deterministic vacation of constant length with a certain probability or an all-inclusive vacation of random period following a general distribution with a different probability. In this scenario, the server's choice may include his option to take no vacation at all with a certain probability.

We find impressive steady-state results in terms of generating functions fro different states of the system. The results for a few unusual cases have been derived.

\section{DESCRIPTION OF THE MATHEMATICAL MODEL}

- We assume single Poisson arrivals with mean arrival rate $\boldsymbol{\omega}(>\mathbf{0})$

- The service time ' $S$ ' follows a general distribution. Let $\boldsymbol{B}(\boldsymbol{x})$ and $\boldsymbol{b}(\boldsymbol{x})$ respectively be the distribution function and the density function of the service time $S$ and let $\mu(x) d x$ be the conditional probability of completion of service, given that the elapsed time is $x$, so that

- $\quad \mu(x)=\frac{b(x)}{1-B_{(x)}}$ and, therefore, $\mathrm{b}(\mathrm{x})=\mu(x) e^{-\int_{0}^{x} \mu(t) d t}$ 
- The server is subject to random failures. Let $\alpha d t$ be the probability that a breakdown will occur during the short time interval $(t, t+d t]$.

- We assume that a breakdown can occur only when the server is proving service and not when it is idle.

- We assume that the customer whose service is interrupted because of a sudden failure returns at the head of the queue and waits until the repairs of the server are complete.

- We assume that as the result of a breakdown, the server undergoes repair process immediately after the occurrence of a failure. When a breakdown occurs, the repair time ' $\mathrm{R}$ ' follows a general distribution. Let $H(x)$ and $h(x)$ respectively be the distribution function and the density function of the repair time $\mathrm{R}$ and let $\theta(x) d x$ be the conditional probability of completion of repairs, given that the elapsed time is $\mathrm{x}$, so that

- $\theta=\frac{h(x)}{H(x)} \quad$ and, therefore, $\theta(x)=h(x) e^{-\int_{0}^{x} \theta(t) d t}$

- After completion of each service, the server has a choice of taking one of the two types of vacations with probability $\delta$, or with probability $1-\delta$, the server may continue staying in the system. We further assume that the server has the choice of taking a type 1 vacation of random length following a general distribution with probability $p_{1}$ or a type 2 vacation of constant duration d with probability $p_{2}, p_{1}+p_{2}=1$.

- Let $G(x)$ and $g(x)$ respectively be the distribution function and the density function of the vacation time V and let $\vartheta(x) d x$ be the conditional probability of completion of a vacation, given that the elapsed time is $\mathrm{x}$, so that

$$
\vartheta(x)=\frac{g(x)}{1-G(x)} \text { and, therefore, } \vartheta(x)=g(x) e^{-\int_{0}^{x} \vartheta(t) d t}
$$

- We assume that on completion of a repair or completion of either type of vacation, the server instantly takes up a customer (at the head of the queue) for service if there is a customer waiting in the queue. However, if on returning the server finds the queue empty, the server still joins the system and remains idle until a new batch of customers arrives in the system.

- Various stochastic processes involved in the system are independent of each other

\section{DEFINITIONS AND EQUATIONS}

We define the following probabilities:

$W_{m}(x, t)$ : probability that at time $t$ there are $\mathrm{n}(\geq 0)$ customers in the queue excluding one customer in service with elapsed service time $x$. Accordingly, $W_{m}(t)=\int_{n=0}^{\infty} W_{m}(x, t)$ denotes the probability that at time t there are $n \geq 0$ customers in the queue excluding one customer in service irrespective of the value of $\mathrm{x}$.

$R_{m}(x, t)$ : probability that at time $\mathrm{t}$ there are $n \geq 1$ customers in the queue and the server is under repairs with elapsed repair time x. .Accordingly, $R_{n}(t)=\int_{0}^{\infty} R_{n}(x, t) d x$ denotes the probability that at time there are $n \geq 1$ customers in the queue and the server is under repairs irrespective of the value of $x$.

$V_{m}^{(1)}(x, t):$ probability that at time $t$ there are $n(>0)$ customers in the queue and the server is on type 1 vacation with elapsed vacation time $x$. deterministic repair time. Accordingly, $V_{m}^{(1)}(t):=\int_{0}^{\infty} V_{m}^{(1)}\left(x_{s} t\right) d x$ denotes the probability that at time $t$ there are $n(>0)$ customers in the queue irrespective of the state of the system.

$V_{m}^{(2)}(t)$ : probability that at time $t$, the server is on type 2 vacation with deterministic vacation time.

$P_{n}(t)=W_{n}(t)+R_{n}(t)+V_{n}^{(1)}(t)+V_{n}^{(2)}(t)$ : probability that at time $t$ there are $\mathrm{n}(\geq 0)$ customers in the queue irrespective of the state of the system.

$\mathrm{Q}(\mathrm{t})$ : probability that there is no customer in the system and the server is idle.

Now, if the steady sate exists, we define the following limiting probabilities as the steady state corresponding to the probabilities defined above for the various states of the system: 
$\operatorname{Lim}_{t \rightarrow \infty} W_{n}(x, t)=W_{n}(x), \operatorname{Lim}_{t \rightarrow \infty} W_{n}(t)=W_{n}, \operatorname{Lim}_{t \rightarrow \infty} R_{m}(x, t)=R_{m}(x), \operatorname{Lim}_{t \rightarrow \infty} R_{n}(t)=R$,

$\operatorname{Lim}_{t \rightarrow \infty} V_{n}^{(1)}(x, t)=V_{n}^{(1)}(x), \operatorname{Lim}_{t \rightarrow \infty} V_{n}^{(1)}(t)=V_{n}^{(1)}, \operatorname{Lim}_{t \rightarrow \infty} V_{n}^{(2)}(t)=V_{n}^{(2)}$.

We further assume that $K_{r}$ is the probability of $\mathrm{r}$ arrivals during the type 1 vacation period and therefore,

$K_{r}=\frac{\exp (\lambda d)(\lambda d)^{r}}{r !} \quad, \quad r=0,1,2, \ldots$

Next, we define the following Probability Generating Functions (PGFs)

$W(x, z)=\sum_{n=0}^{m} W_{n}(x) z^{n}, W(z)=\sum_{n=0}^{m} W_{n 1} z^{n}$,

$R(x, z)=\sum_{n=1}^{\infty} R_{n}(x) z^{n}, R(z)=\sum_{n=1}^{\infty} R_{n} z^{n}$,

$V^{(1)}(x, z)=\sum_{n=0}^{\infty} V_{n}^{(1)}(x) z^{n}, V^{(1)}(z)=\sum_{n=0}^{\infty} V_{n}^{(1)} z^{n}$,

$V^{(2)}(z)=\sum_{n=0}^{\infty} V_{n}^{(2)} z^{n},(3.6)$

$\left.K(z)=\sum_{n=0}^{\infty} K_{n} z^{n}=\sum_{n=0}^{\infty e} \frac{e^{-\omega d}(\omega d)^{n}}{n !}=e^{-\omega d[1-z)}, \mid z\right\}<1$.

\section{STEADY STATE EQUATIONS}

Applying the usual probability arguments based on the underlying assumptions, we obtain the following steady state equations:

$\frac{d}{d x} W_{m}(x)+(\omega+\mu(x)+\alpha) W_{m}(x)=\omega W_{m-1}(x), n \geq 1$,

$\frac{d}{d x} W_{0}(x)+(\omega+\mu(x)+\alpha) W_{0}(x)=0$,

$\frac{\mathbb{d}}{\mathbb{d} x} R_{n}(x)+(\omega+\theta(x)) R_{m}(x)=\omega R_{m-1}(x), n \geq 2$

$\frac{d}{d x} R_{1}(x)+(\omega+\theta(x)) R_{1}(x)=0$,

$\frac{d}{d x} V_{n}^{(1)}(x)+(\omega+\theta(x)) V_{n}^{(1)}(x)(x)=\omega V_{m-1}^{(1)}(x)(x), n \geq 1$,

$\frac{d}{d x} V_{0}^{(1)}(x)+(\omega+\theta(x)) w_{0}(x)=0$,

$V_{n}^{(2)}=\delta p_{2} \int_{0}^{\infty} W_{n}(x) \mu(x) d x, n \geq 0$,

$\omega Q=(1-\delta) \int_{0}^{m \infty} W_{0}(x) \mu(x) d x+\int_{0}^{\infty 8} V_{0}^{(1)}(x) \vartheta(x) d x+V_{0}^{(2)} k_{0}$.

The above equations would be solved subject to the following boundary conditions:

$W_{m}(0)=(1-\delta) \int_{0}^{\infty} W_{m+1}(x) \mu(x)+\int_{0}^{x} V_{m+1}^{(1)}(x) \vartheta(x) d x+\int_{0}^{\infty} R_{m+1}(x) \theta(x) d x$,

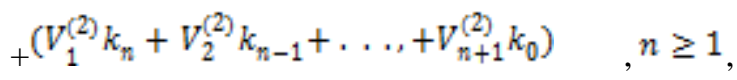


$W_{0}(0)=(1-\delta) \int_{0}^{\infty} W_{1}(x) \mu(x)+\int_{0}^{x} V_{1}^{(1)}(x) \vartheta(x) d x_{+} \int_{0}^{\infty} R_{1}(x) \theta(x) d x$,

$+\left(V_{1}^{(2)} k_{0}+V_{0}^{(2)} k_{1}\right)$

$R_{m+1}(0)=\propto W_{n}, \quad n \geq 0$,

$V_{n}^{(1)}(0)=\delta p_{1} \int_{0}^{\infty} W_{n}(x) \mu(x) d x, n \geq 0$.

\section{STEADY STATE SOLUTION}

Applying the usual solution methods under the generating function approach, we obtain the following results:

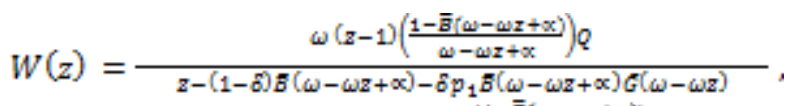

$$
\begin{aligned}
& -\partial p_{2} K(z) B(\omega-\omega z+x)-x\left(\frac{1-\bar{b}(\omega-\omega x+x)}{w-w I+x}\right) H(\omega-\omega z) \\
& R(z)=\frac{\alpha\left(\frac{1-\overline{\bar{E}}(\omega-\omega x+x)}{\omega-\omega x+x}\right)(R(\omega-\omega z)-1) Q}{z-(1-b) B(\omega-\omega z+\alpha)-\delta p_{1} E(\omega-\omega z+x) G(\omega-\omega z)} . \\
& -6 p_{2} K(z) E(\omega-\omega z+\alpha)-\alpha\left(\frac{1-\bar{B}(\omega-\omega x+x)}{\omega-\omega x+x}\right) R(\omega-\omega z)
\end{aligned}
$$

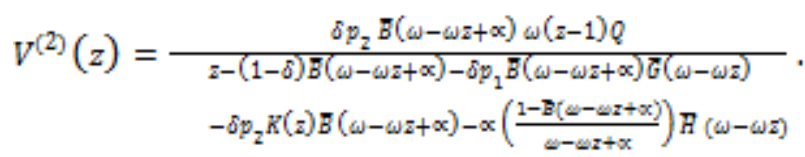

Next, using the normalizing condition

$Q+W(1)+R(1)+V^{(1)}(1)+V^{(2)}(1)=1$.

We obtain

$$
Q==\frac{\begin{array}{c}
1-\delta p_{1} \bar{B}(\propto) \omega E(V)-\delta p_{2} \bar{B}(\propto) \omega d \\
\propto\left(\frac{1-\bar{B}(\propto)}{\propto}\right)-(1-\bar{B}(\propto)) \omega E(R)
\end{array} .}{1+\delta p_{2} \bar{B}(\propto) \omega(1-d)} .
$$

Note that the result (5.6) gives the probability that the server is idle and the stability condition that emerges from this result is given by

$\delta p_{1} \bar{B}(\propto) \omega E(V)+\delta p_{2} \bar{B}(\propto) \omega d$

$+\omega\left(\frac{1-\bar{B}(\propto)}{\propto}\right)+(1-\bar{B}(\propto)) \omega E(R)<1$. 
Next, on substituting the value of $Q$ from (5.6) into equations

(5.1), (5.2), (5.3) and (5.4) we obtain

$W(1)=\frac{\omega\left(\frac{1-\bar{B}(\propto)}{\propto}\right)}{1+\delta p_{2} \bar{B}(\propto) \omega(1-d)^{\prime}}$

This is the steady stae probability that the server is busy providing service to customers.

$R(1)=\frac{\propto \omega\left(\frac{1-\bar{B}(\propto)}{\propto}\right) E(R)}{1+\delta p_{2} \bar{B}(\propto) \omega(1-d)}$.

This is the steady-state probability that the server is under repairs.

$V^{(1)}(1)=\frac{\delta p_{1} \omega \bar{B}(\propto) E(V)}{1+\delta p_{2} \bar{B}(\propto) \omega(1-d)}$.

This is the steady-state probability that the server is on type 1 general vacation

$V^{(2)}(1)=\frac{\delta p_{2} \omega \bar{B}(\propto)}{1+\delta p_{2} \bar{B}(\propto) \omega(1-d)}$

This is the steady probability that the server is under type 2 vacation of constant duration.

\section{PARTICULAR CASES}

The following interesting cases can be obtained as follows:

\section{Case 1: Breakdowns and Only Type 1 Server Vacations}

We can put $p_{1}=1$ and $p_{2}=0$ in the main results found above.

\section{Case 2: Breakdowns and Only Type 2 Vacations}

In this case, we substitute $p_{1}=0$ and $p_{2}=1$ in the main results.

\section{Case 3: Breakdowns and No Vacations}

In this case, we substitute $\delta=0$ in the main results. 
In this case, we substitute $\propto=0$ in the results of case 1 .

Consequently, we substitute $\bar{B}(0)=1$ and $\lim _{\mathrm{x} \rightarrow 0}\left(\frac{1-\mathbb{B}_{[}(\mathrm{x})}{\mathrm{x}}\right)=\mathrm{E}(\mathrm{S})$,

where $\mathrm{E}(\mathrm{S})$ is the mean service time.

\section{Case 5: No Breakdowns and Only Type 2 Vacations}

In this case, we substitute ${ }^{\propto=0}$ in the results of case 2 .

\section{Case 6: No Breakdowns and No vacations}

In this case, we substitute ${ }^{\propto=0}$ in the results of case 3 .

\section{CONCLUSION}

In this paper we studied a new model of a queueing system which is subject to random breakdowns as well as server vacations. The latest significant assumptions in the article are that the server has the option to take a vacation on completing every service and in addition, if the server wishes to take a vacation, then he has the opportunity to take a deterministic vacation of constant length as it happens in some organizations who offer a fixed-length break, i. e. a lunch break to its employees or a general vacation of random length as it happens in many systems where the senior manager or a managing director may like to take a vacation of uncertain duration in order to attend to other essential jobs

We have found the essential meaningful steady-state results in terms of probability generating functions in equations (5.1) to (5.6), the critical steady-state condition under which the steady-state exists in equation (5.7) and the steady-state probabilities of various states of the system in equations (5.8) to (5.11). Many meaningful and exciting results are derived cases 1 to 6 . Hopefully, these new results will be an excellent addition to the literature of queueing theory.

\section{REFERENCES}

[1] Gaver, D.P. (1962): A Waiting Line with Interrupted Service Including Priorities. Journal of Royal Statistical Society, Ser. - $B, 24$ (1962), 73-90.

[2] Madan, Kailash C. (1999): An M/G/1 Queue with optional deterministic server vacations. Metron, LVII No. 3-4 (1999) 83-95.

[3] Madan, Kailash C. (2011): 'A non-preemptive priority queueing system with a single server serving two queues M/G/1 and $\mathrm{M} / \mathrm{D} / 1$ with Optional server vacations based on exhaustive service of the priority units. Applied Mathematics, Vol. 3, No. 1, pp. 03-08.

[4] Madan, Kailash C. (2015): On a $\mathrm{M}(\mathrm{X}) /(\mathrm{G} 1, \mathrm{G} 2) / 1$ queue with third stage optional service and deterministic server vacations. Journal of Mathematical and Computational Science, Vol. 5, No. 2, pp. 195-206.

[5] Takagi, H. (1992): Time dependent process of M/G/1 vacation models with exhaustive service. J. Appl. Prob., 29, 418429.

[6] Tegham, L.J. (1990): On a decomposition result for a class of vacation queueing systems. Journal of Applied Probability $27,227-231$. 